\title{
Increased circulating nerve growth factor is directly correlated with disease activity in juvenile chronic arthritis
}

University of Florence, Florence, Italy: Department of Paediatrics

F Falcini

M Ermini

Institute of Internal Medicine IV

M Matucci Cerinic

A Lombardi

$S$ Generini

A Pignone

Consiglio Nazionale delle Ricerche, Institute of

Neurobiology, Rome,

Italy

P Tirassa

L Aloe

Department of Paediatrics, IRCCS Burlo Garofalo, University of Trieste, Trieste, Italy

L Lepore

Ludwig Boltzmann

Institute of

Rheumatology, Vienna,

Austria

G Partsch

Correspondence to: Fernanda Falcini MD, Department of Paediatrics, via Lungo L'Africo 82/B, 50137 Florence, Italy.

Accepted for publication 7 June 1996

F Falcini, M Matucci Cerinic, A Lombardi, S Generini, A Pignone, P Tirassa, M Ermini, L Lepore, G Partsch, L Aloe

\begin{abstract}
Objective-To determine the circulating serum concentrations of nerve growth factor (NGF) and compare them with indices of disease activity in juvenile chronic arthritis.

Methods-NGF concentrations were evaluated with a two site immunoenzymatic assay (ELISA), in 17 children with systemic, 39 with polyarticular, and 24 with pauciarticular onset juvenile chronic arthritis. Each subset was divided according to different variables, appropriate to each subset, reflecting active and inactive disease.

Results-NGF concentrations were significantly higher in children with systemic [254 (SD 256.1) $\mathrm{pg} \mathrm{ml}^{-1} ; P<0.001$ ], polyarticular [165.2 (300.8) $\left.\mathrm{pg} \mathrm{ml}^{-1} ; P<0.05\right]$, and pauciarticular $\left[106.8(111.8) \mathrm{pg} \mathrm{m}^{-1} ; \mathrm{P}<\right.$ $0.005]$ onset juvenile chronic arthritis than in controls. In all subsets, NGF concentrations were higher in the active than in the inactive phase of the disease. A significant direct correlation between NGF concentrations and erythrocyte sedimentation rate was found both in the systemic and in the polyarticular onset juvenile chronic arthritis.
\end{abstract}

Conclusions-The increase in NGF concentrations in all juvenile chronic arthritis subsets and the correlation with disease activity suggest that NGF may take an active part in joint inflammation.

\section{(Ann Rheum Dis 1996;55:745-748)}

Nerve growth factor (NGF) is a neurotrophic protein responsible for the development and differentiation of sympathetic, sensory, and cholinergic neurones. ${ }^{1}$ In the peripheral nervous system, NGF is involved in the modulation of the function of the nerve terminals, controlling their development, survival, and production and release of neurotransmitters. ${ }^{1}$ NGF is produced by different cells, such as keratinocytes, smooth muscle cells, fibroblasts, and lymphocytes. ${ }^{1}$

NGF has been shown to increase the chemotaxis of polymorphonuclear cells, the B cell production of immunoglobulins, and the $T$ cell dependent production of autoantibodies. ${ }^{2}$ NGF interacts specifically with nociceptive sensory neurones, by increasing the content of neuropeptides, in particular substance $\mathrm{P}^{3}$ The release of neuropeptides from nerve terminals elicits a local flare called neurogenic inflammation $^{4}$ mainly linked to the release of substance $P$ which is involved in the activation of synoviocytes and cartilage breakdown in arthritis (for review see Matucci-Cerinic, $\left.1993^{5}\right)$. The presence of NGF is essential in the production and in particular the release of neuropeptides induced by different stimuli. ${ }^{6}$ This evidence suggests that the presence of NGF is associated with the maintenance of neurogenic inflammation. ${ }^{4}$ Previous studies showed that NGF is increased in diseases with an immune pathogenesis, such as multiple sclerosis, ${ }^{7}$ systemic lupus erythematosus (SLE) ${ }^{89}$ systemic sclerosis (unpublished data), and, in particular, Kawasaki's disease ( $F$ Falcini et al, unpublished data). In adult rheumatoid arthritis, high NGF concentrations have been reported in synovial fluid and synovial membrane ${ }^{10}$ and in serum. ${ }^{9}$

Increased NGF concentrations have also been found in the joint tissues of rats with adjuvant arthritis, ${ }^{11}$ and in arthritic transgenic mice carrying and expressing the human tumour necrosis factor $\alpha$ (TNF- $\alpha$ ) gene. ${ }^{12}$

Juvenile chronic arthritis is a chronic inflammatory disease characterised by articular and systemic involvement in which three types of onset are recognised: systemic, polyarticular, and pauciarticular. ${ }^{13}$ As far as we know, there are no published studies concerning the role of the peripheral nervous system and neurogenic inflammation in juvenile chronic arthritis, and no studies evaluating NGF, either in the synovium and synovial fluid or in circulation, in this condition are available. High concentrations of NGF were found in the synovial fluid of one child affected with juvenile chronic arthritis. ${ }^{10}$ To date, NGF concentrations have not been investigated in a large population of children with juvenile chronic arthritis and this prompted us to investigate serum NGF and to evaluate its possible role in the genesis of inflammation by comparing it with clinical and haematological indices of disease activity.

\section{Methods}

PATIENTS

Eighty children ( 54 girls and 26 boys), aged from 15 months to 14 years (mean age 7 years 8 months), all fulfilling EULAR criteria for the diagnosis of juvenile chronic arthritis, ${ }^{14}$ were selected and entered the study. Seventeen had systemic (age 5 to 12 years), 39 polyarticular (age 3 to 4 years), and 24 pauciarticular onset (age 11 months to 4 years). The duration of the 
disease ranged from 8 months to 12 years (mean duration 8 years, 9 months). Each subset was then divided according to the clinical findings (fever, rash, joint inflammation) and haematological data, erythrocyte sedimentation rate (ESR), and $\mathrm{C}$ reactive protein into two groups, one with active and the other with inactive disease. No patient was in remission.

Systemic juvenile chronic arthritis was defined active in presence of all the following variables: spiking fever $>38.5^{\circ} \mathrm{C}$; typical rash; joint swelling with reduced range of motion in one or more joints; $\mathrm{Hb}<10 \mathrm{~g} \mathrm{dl}^{-1} ; 5$. ESR $>25$ $\mathrm{mm} \mathrm{h}^{-1}$.

Polyarticular arthritis was defined active in the presence of: (1) ESR > $25 \mathrm{~mm} \mathrm{~h}^{-1}$; (2) swelling with reduced range of motion in more than five joints. Pauciarticular arthritis was defined active in presence of joint swelling with reduced range of motion in one to four joints.

The basic treatment in children with systemic juvenile chronic arthritis consisted of steroids and non-steroidal anti-inflammatory drugs (NSAID), while in polyarticular subset, methotrexate represented the second line drug used in association with NSAID. All patients in the pauciarticular onset group were given only NSAID. Forty healthy children (25 girls, 15 boys; mean age 6 years, 7 months) matched for sex and age were chosen as controls.

Disease activity was scored following a previous scoring system modified by $\mathrm{us}^{15}: 0=$ no activity; systemic subset $1=$ fever $<38^{\circ} \mathrm{C}$, mild joint swelling, ESR $25-40 \mathrm{~mm} \mathrm{~h}^{-1}, \mathrm{Hb}$ 9-10 $\mathrm{g} \mathrm{dl}^{-1} ; 2=$ fever $<39^{\circ} \mathrm{C}$, moderate joint swelling, ESR 40-60 mm h${ }^{-1}, \mathrm{Hb} \mathrm{8-9} \mathrm{g} \mathrm{dl}^{-1} ; 3=$ fever $>39^{\circ} \mathrm{C}$, severe joint swelling, ESR $>60$ $\mathrm{mm} \mathrm{h}^{-1}, \mathrm{Hb} 6-8 \mathrm{~g} \mathrm{dl}^{-1}$; polyarticular subset $1=$ mild joint swelling, ESR $25-40 \mathrm{~mm} \mathrm{~h}^{-1} ; 2=$ moderate joint swelling, ESR $40-60 \mathrm{~mm} \mathrm{~h}^{-1} ; 3$ = severe joint swelling, ESR $>60 \mathrm{~mm} \mathrm{~h}^{-1}$; pauciarticular subset $1=$ mild, $2=$ severe joint swelling.

\section{PROCEDURES}

After consent had been obtained from the parents, blood was drawn during routine haematological analysis in the morning between 8 am and 9 am and quickly centrifuged. Sera were then stored at $-70^{\circ} \mathrm{C}$ until used for NGF determination. NGF was prepared from mouse salivary glands following the procedure of Bocchini and Angeletti ${ }^{16}$ and a monoclonal antiNGF antibody, which recognises rodent and human NGF, was prepared in our laboratory. The NGF content in human sera was measured by a two site immunoenzymatic assay (ELISA). Briefly, 96-well polystyrene microtitre immunoplates (Nunc) were coated with affinity purified polyclonal goat anti-NGF antibody. Parallel wells were coated with affinity purified polyclonal goat IgG for evaluation of the non-specific signal. After $5 \mathrm{~h}$ at $20^{\circ} \mathrm{C}$, the plates were washed (as in subsequent steps) with phosphate buffered saline (PBS) / $0.0005 \%$ Tween $20 / 1 \%$ fetal calf serum (FCS) to block non-specific binding sites. After washing, the NGF standard solutions, ranging from 0 to $1 \mathrm{ng} \mathrm{ml}^{-1}$, were distributed in each plate. The samples were ultrasonicated at $4^{\circ} \mathrm{C}$ in the sample buffer and centrifuged at $6700 \mathrm{~g}$ for $30 \mathrm{~min}$ at $4^{\circ} \mathrm{C}$. A $100 \mu \mathrm{l}$ aliquot of supernatant was added to each well and the plates incubated overnight at $4^{\circ} \mathrm{C}$. The plates were then washed and $100 \mu \mathrm{l}$ of monoclonal antibody against NGF, diluted 1:100 in PBS, were added to each well. After incubation for 4 $h$ at $20^{\circ} \mathrm{C}$, the plates were washed and incubated with biotinylated rat immunoglobulins (1:8000, Zymed). The subsequent incubation with peroxidase conjugated streptavidin (Zymed) and addition of ortophenyldiamine resulted in a colorimetric reaction the optical density of which was measured at $490 \mathrm{~nm}$ using a Dynatech MR 5000 microplate reader. Specificity for NGF was also assessed using a recombinant human NGF. ${ }^{72}$ Recombinant brain derived neurotrophic factor is not recognised in the ELISA at concentrations up to $20 \mathrm{ng}$ $\mathrm{ml}^{-1}$.

Serum NGF was correlated with disease activity and haematological variables (that is, ESR and $C$ reactive protein).

\section{STATISTICAL ANALYSIS}

Evaluation of the differences in NGF levels observed between the three groups of juvenile chronic arthritis and controls was performed with the unpaired Student $t$ test. The significance of the differences was also confirmed by the parameter-free MannWhitney $U$ test. The correlation between NGF and ESR, C reactive protein, and IgG, in both active and inactive disease, was tested by Pearson's correlation coefficient.

\section{Results}

Of the 80 children studied, 53 were in the active phase of the disease and 27 in the inactive phase. The mean NGF value in healthy children was 6 (SD 4.5) $\mathrm{pg} \mathrm{m}^{-1}$. The results show significantly raised concentrations of NGF in children with systemic juvenile chronic arthritis [254 (256.1) $\mathrm{pg} \mathrm{ml}^{-1} ; \mathrm{P}<$ 0.001] than in controls, in both the active phase [259.3 (280.4) $\left.\mathrm{pg} \mathrm{ml}^{-1} ; \mathrm{P}<0.001\right]$ and the inactive phase $\left[91.6(87.2) \mathrm{pg} \mathrm{ml}^{-1} ; \mathrm{P}<\right.$ 0.001 ] of the disease. NGF concentrations were raised in all patients with active disease and-although at a lesser extent-also in the inactive phase of the disease. In the polyarticular subset, NGF values were significantly increased in the whole group [165.2 (300.8) pg $\left.\mathrm{ml}^{-1} ; \mathrm{P}<0.05\right]$, and were always higher in the active phase [223.9 (347.1) $\mathrm{pg} \mathrm{ml}^{-1}$ ] than in the inactive phase $\left[33.3(16.2) \mathrm{pg} \mathrm{ml}^{1}\right]$ of the disease. The mean concentration of NGF in all children with pauciarticular arthritis was increased [106.8 (111.8) $\left.\mathrm{pg} \mathrm{ml}^{-1} ; \mathrm{P}<0.005\right]$; in the active phase NGF was also raised, at 151.7 (124.5) $\mathrm{pg} \mathrm{ml}^{-1}$, though to a lesser degree than in the other two subsets; patients with inactive disease had higher serum NGF values than the healthy controls $\left[39.6(28.8) \mathrm{pg} \mathrm{ml}^{-1}\right]$.

In each subset, a statistical correlation was performed between serum NGF and ESR, C reactive protein, and the activity scores. In systemic onset, a very significant correlation was found with $\operatorname{ESR}(r=0.9 ; \mathrm{P}<0.001)$ and $\mathrm{C}$ reactive protein $(r=0.8 ; \mathrm{P}<0.001)$. In polyar- 


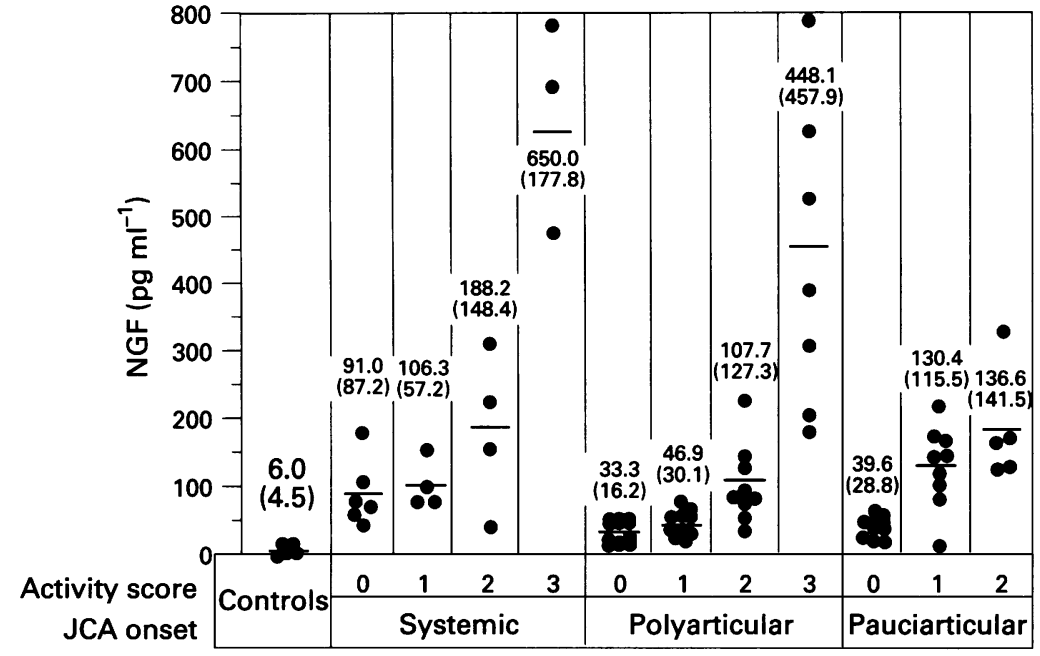

Figure 1 Nerve growth factor (NGF) concentrations ( $p g \mathrm{ml}^{-1}$ ) detected for each subgroup of juvenile chronic arthritis (FCA) in different grades of disease activity.

ticular onset, there was a significant correlation in active and inactive disease with ESR ( $r=$ $0.4 ; \mathrm{P}<0.05)$ but not with $\mathrm{C}$ reactive protein $(r=0.3$, NS). No correlation was found with ESR and $C$ reactive protein in pauciarticular onset.

Correlation of NGF with the different grade of disease activity in each subset showed that NGF concentrations rise progressively in parallel with the increase in disease activity (fig 1). The increase was more significant when the disease reached activity grades 2 and 3 .

NGF concentrations were also determined in synovial fluid from two children with active pauciarticular juvenile chronic arthritis (376 and $309 \mathrm{pg} \mathrm{ml}^{-1}$; serum concentrations were 155 and $158 \mathrm{pg} \mathrm{ml}^{-1}$ ) respectively.

\section{Discussion}

Our data show a striking rise in circulating NGF in juvenile chronic arthritis. The highest values are detected in systemic onset juvenile chronic arthritis, but in the other two subsets the values are higher than in controls. NGF concentrations are increased in the active

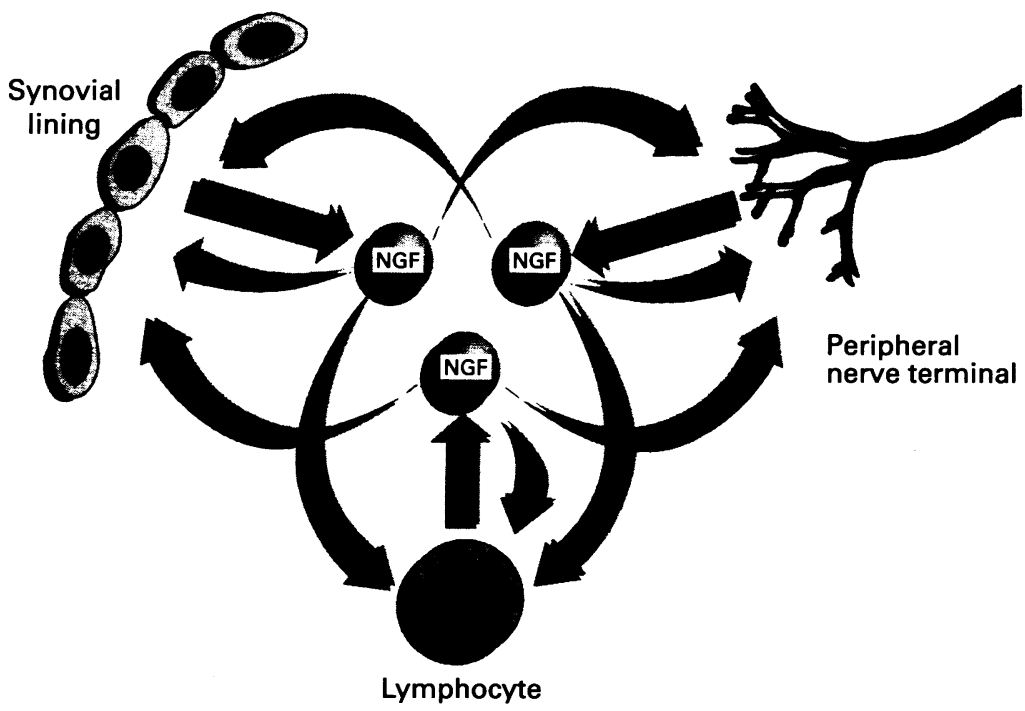

Figure 2 The possible sources of nerve growth factor (NGF) are shown. It is noteworthy that NGF may act in an autocrine and paracrine way. It may be released by nerve

terminals and influence the immune and synovial systems, as well as being released by those two systems, which influence each other and the nervous system. phase of each subset and remain raised in the inactive phase indicating also that if disease activity is decreasing the serum NGF is kept at a higher level by mechanism still to be determined. NGF concentrations are also high in the pauciarticular subset of juvenile chronic arthritis, where the inflammatory process is limited to one or few joints and systemic involvement is usually lacking.

The source of the increased circulating concentrations of NGF is still a matter of debate (fig 2). The local increase may be derived from synoviocyte proliferation or lymphocytes or peripheral nervous terminals activation in inflamed joints, or a combination of all these. Some pathogenic events (for example, local production of TNF- $\alpha$ and interleukin 1) may trigger the significant increase of NGF in synovial fluid and in the systemic circulation. Cytokines such as interleukin 1 , interleukin 6 , and TNF- $\alpha$ play an important role in the pathogenesis of juvenile chronic arthritis. ${ }^{151718}$ These cytokines have been shown to stimulate NGF synthesis by the peripheral nervous system during chronic inflammatory processes. ${ }^{19} \mathrm{NGF}$ is produced by peripheral nerve endings, ${ }^{1}$ fibroblasts, ${ }^{20}$ and lymphocytes ${ }^{21}$ (see fig 2), and the interleukin 1 and TNF- $\alpha$ dependent stimulation of these cells might be related-as potential alternative sources-to the striking increase in the protein in the serum of systemic onset patients. This hypothesis may be supported by the evidence that in systemic subsets of juvenile chronic arthritis, where constitutional findings such as fever are prominent and joint involvement is less relevant, we detected the highest circulating NGF concentrations. In this case, the production of NGF by lymphocytes may also have a particular role in the disease pathogenesis. $\mathrm{B}$ and $\mathrm{T}$ lymphocytes possess the Trk protein family on their membrane, ${ }^{22}$ serving as signal transducing receptor unit for NGF. They may favour the activity of NGF as an autocrine protein, thus enhancing an amplificatory loop involving macrophages and lymphocytes-fundamental cells for the development and maintenance of juvenile chronic arthritis. ${ }^{13}$

On the other hand, local production (that is, by synoviocytes) might account for the presence of high NGF values in polyarticular subset where fever is rarely present, indicating mild systemic involvement, and in pauciarticular onset, where systemic findings are absent. Another point that should be stressed is the possible participation of NGF in pain transmission. It has been shown that cleaved NGF alters pain threshold in injured target regions of NGF responsive neurones. ${ }^{23}$ This property might suggest that increased NGF concentrations may enhance the sensitivity to pain of the peripheral terminals of primary afferent nociceptors involved in joint inflammation in juvenile chronic arthritis. NGF is retrogradely transported by sensory nerves as well as by sympathetic nerves and has been shown to increase neuropeptide content in adult sensory neurones. ${ }^{24}$

NGF induces the synthesis and the release from sensory endings of substance $P$, which is 
mainly related to neurogenic inflammation. ${ }^{34}$ The presence of NGF is considered to be the main switch to activate the release of neuropeptides from $\mathrm{C}$ fibres and generate neurogenic inflammation. ${ }^{6}$ In this perspective, the increased concentrations of NGF, either in the circulation or in synovial fluid, might amplify the inflammatory joint process by facilitating the development of neurogenic inflammation.

It is interesting to stress that NGF concentrations are directly correlated to ESR and in particular to the progressive increase in disease activity. This kind of behaviour is similar to that observed in multiple sclerosis, where NGF increases during acute attacks and diminishes during remission. ${ }^{7}$ In SLE, increased NGF concentrations have been found concomitantly with disease relapses. ${ }^{8}$

Our data clearly show that NGF concentrations are increased in all juvenile chronic arthritis subsets and are correlated with disease activity. This evidence seems to strengthen the hypothesis that NGF is involved-primarily or secondarily - in inflammation, though it is still unknown whether it plays an active role or is just the result of a defence mechanism in the pathogenesis of juvenile chronic arthritis. Further investigations are needed to determine the source of circulating NGF, to discover whether NGF is a marker of disease activity, and to identify its real significance in the evolution of the disease.

This work was supported by a grant of "Ministero della Ricerca Scientifica", quota $60 \%$.

1 Levi Montalcini R. Nerve growth factor: 35 years later. Science 1987;164:245-52.

2 Otten U, Ehrard P, Peck R. Nerve growth factor induces growth and differentiation of human B lymphocytes. Proc growth and differentiation of human B
Natl Acad Sci USA 1989;86:10059-63.

3 Otten U, Lorenz HP. Nerve growth factor increases substance $P$, cholecistokinin and vasoactive intestinal polypeptide immunoreactivities in primary sensory neurones of newborn rats. Neurosci Lett 1983;4:153-8.

4 Foreman JC, Jordan CC. Neurogenic inflammation. Trends Pharmacol Sci 1984;5:1 16-9.

5 Matucci Cerinic M. Sensory neuropeptides and rheumatic diseases. Rheum Dis Clin North Am 1993;19:975-91.
6 Miller MS, Buck SH, Sipes IG, Yamamura HI, Burks TF. Regulation of substance P by NGF: dysruption by capsaicin. Brain Res 1987;250:193-6.

7 Bracci Laudiero M, Aloe L, Levi Montalcini R, Buttinelli C, Schilter D, Gillesen S, et al. Multiple sclerosis patients express increased levels of b-nerve growth factor in cerebrospinal fluid. Neurosci Lett 1992;147:9-12.

8 Bracci Laudiero L, Aloe L, Levi Montalcini R, Galeazzi M, Schilter D, Scully JL, Otten U. Increased levels of NGF in sera of systemic lupus erythematosus patients. Neuroreport 1993;4:563-5.

9 Dicon E, Masson C, Jabbour W, Nerviere V. Increased frequency of NGF in sera of rheumatoid arthritis and systemic lupus erythematosus patients. Neuroreport 1993; 5:321-4.

10 Aloe L, Tuveri MA, Carcassi U, Levi Montalcini R. Nerve growth factor in the synovial fluid of the patients with growth factor in the synovial fluid of the patient
chronic arthritis. Arthritis Rheum 1992;35:351-5.

11 Aloe L, Tuveri MA, Levi Montalcini R. Studies on carragenan-induced arthritis in adult rats: presence of NGF and role of sympathetic innervation. Rheumatol Int 1992;12:213-6.

12 Aloe L, Probert L, Collias G, Bracci Laudiero L, Spillantini MG, Levi Montalcini $R$. The synovium of transgenic arthritic mice expressing human necrosis factor contains a high level of nerve growth factor. Growth Factors 1993;9:149-55.

13 Cassidy JT, Petty RE. Juvenile rheumatoid arthritis. In: JT Cassidy, RE Petty, eds. Textbook of pediatric rheumatology, 3rd ed. Philadelphia: WB Saunders, 1995:133-223.

14 Wood PHN. Special meeting on nomenclature and classification of arthritis in children. In: Munther M, ed. The care of rheumatic children. Basel: EULAR, 1978.

15 Rooney M, David J, Symons J, Di Giovine F, Varsani H, Woo P. Inflammatory cytokine responses in juvenile chronic arthritis. Br F Rheumatol 1995;34:454-60.

16 Bocchini G, Angeletti PU. The nerve growth factor: purification as 30.000 molecular weight protein. Proc Natl Acad cation as 30.000 molecular
Sci USA 1969;64:787-94.

17 Martini A, Ravelli A, Notarangelo LP. Enhanced interleukin 1 and depressed interleukin 2 production in juvenile arthritis. $\mathcal{F}$ Rheumatol 1986;13:588-92.

18 Brennan MF, Maini RV, Feldmann M. TNF a: a pivotal role in rheumatoid arthritis. Br $\mathcal{F}$ Rheumatol 1992;31:293-8.

19 Levi Montalcini R, Aloe L, Alleva E. A role for nerve growth factor in nervous, endocrine and immune systems. Prog Neurol Endocrinol Immunol 1990;1:1-10.

20 Murase K, Murakami Y, Takayanagi K, Furukawa Y, Hayashi K. Human fibroblasts cells synthetize and secrete
nerve growth factor in culture. Biochem Biophys Res Commun $1992 ; 184: 373-9$.

21 Santambrogio N, Benedetti M, Chao MV, Muzaffar R, Kulig K, Gabellini N, et al. Nerve growth factor production by lymphocytes. F Immunol 1994;153:448895.

22 Lindsay RM, Wiegand SI, Altar A, Di Stefano PS. Neurotrophic factors: from molecule to man. Trends Neurol Sci 1994;17:182-90.

23 Taiwo YO, Levine JD, Burch RM, Woo JE, Mobley WC Hyperalgesia induced in the rat by the aminoterminal octapeptide of NGF. Proc Natl Acad Sci USA 1991; 88:5144-8.

24 Stoeckel K, Schweb M, Thoenen H. Specificity of retrograde transport of NGF in sensory neurons: a biochemical and morphological study. Brain Res 1975; 89:1-14. 